MKG-Chirurg 2015 $\cdot 8: 125-126$

DOI 10.1007/s12285-015-0022-5

Online publiziert: 2. September 2015

(c) Springer-Verlag Berlin Heidelberg 2015

CrossMark

\author{
G. Gehrke ${ }^{1} \cdot$ S. Reinert ${ }^{2}$ \\ ${ }^{1}$ Klinik für Mund-, Kiefer- und Gesichtschirurgie, plastische und ästhetische Operationen, \\ Diakoniekrankenhaus Henriettenstiftung gGmbH, Hannover, Deutschland \\ ${ }^{2}$ Klinik und Poliklinik für Mund-, Kiefer- und Gesichtschirurgie, \\ Universitätsklinikum Tübingen, Tübingen, Deutschland
}

\title{
Erkrankungen der Speicheldrüsen
}

gang in die kürzlich erschienene S2k-Leitlinie der AWMF gefunden.

Die gutartigen Speicheldrüsentumoren sind Thema des zweiten Beitrags. Etwa 2\% aller Tumoren des Menschen sind Speicheldrüsentumoren, $80 \%$ davon sind in der Glandula parotis lokalisiert. Die Einteilung der Speicheldrüsentumoren erfolgt nach wie vor nach der aktuellen WHO-Klassifikation aus dem Jahre 2005 in zehn verschiedene benigne und 23 maligne epitheliale Tumoren.

Die Diagnostik stützt sich auf die hochauflösende B-Scan- und Farbduplexsonographie sowie die MRT. Die Feinnadelaspirationszytologie erfordert eine besondere Erfahrung des Pathologen, die Stanzbiopsie gelangt nur in besonderen Fällen zur Anwendung.

In der Therapie des pleomorphen Adenoms hat sich neben der lateralen $\mathrm{Pa}$ rotidektomie unter Präparation des N. facialis die extrakapsuläre Tumorexstirpation als Standardverfahren etabliert. Bei Tumoren im tiefen Lappen medial der Fazialisebene ist ein abgestuftes Vorgehen bis zur vollständigen konservativen Parotidektomie indiziert. In diesen Fällen ermöglicht die Rekonstruktion des fehlenden Gewebes durch einen M.-sternocleidomastoideus-Lappen (Rauch-Plastik) ästhetisch sehr gute Ergebnisse. Die Therapie der anderen Adenome der Glandula parotis orientiert sich weitgehend an diesem Konzept, benigne Tumoren der Glandula submandibularis und der kleinen Speicheldrüsen werden durch vollständige Drüsenexstirpation behandelt.

Der dritte Beitrag ist schließlich den malignen Speicheldrüsentumoren gewidmet, die eine extrem heterogene Gruppe seltener Tumoren mit großer klini- scher, pathologischer und molekularbiologischer Variabilität darstellen. Im Fokus der aktuellen Forschung steht die histologische und neu die molekularbiologische Charakterisierung der Tumoren. So wurden neue Entitäten wie das „mammaanaloge sekretorische Karzinom“ beschrieben, darüber hinaus wurde der Begriff „High-Grade-Transformation“ mit Verlust der tumorspezifischen Histologie geprägt. Ätiopathogenetisch zeigen verschiedene Subtypen wie z. B. das adenoidzystische Karzinom oder das Mukoepidermoidkarzinom spezifische chromosomale Aberrationen, die mit der Bildung von sog. Fusionsgenen einhergehen und für die Ausbildung und das Wachstum der entsprechenden Tumoren verantwortlich gemacht werden. Die chirurgische Resektion, oftmals mit adjuvanter Radio(chemo)therapie, stellt die Therapie der Wahl dar, während herkömmliche Chemotherapeutika kaum eine Wirksamkeit besitzen. Die zunehmende Erkenntnis der genomischen Alterationen und tumorspezifischen Signalwege eröffnet neue vielversprechende Möglichkeiten zielgerichteter molekularer Therapien im fortgeschrittenen Tumorstadium.

Wir wünschen den Lesern eine spannende und informative Lektüre und hoffen, dass die Artikel dazu beitragen können, Anregungen für die Versorgung der uns anvertrauten Patienten und für wissenschaftliche Arbeiten zu geben. z. T. noch nicht definitiv beurteilt werden kann. In der Therapie eröffnet insbesondere die interventionelle Speichelgangendoskopie (iSGE), evtl. kombiniert mit mikrochirurgischen Verfahren, als minimalinvasives Verfahren schonende Behandlungsmöglichkeiten und hat auch Ein- 


\section{Q. Auterer}

Prof. Dr. Dr. G. Gehrke

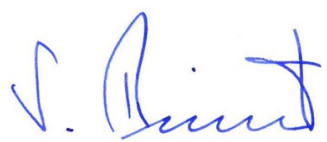

Prof. Dr. Dr. S. Reinert

\section{Korrespondenzadressen}

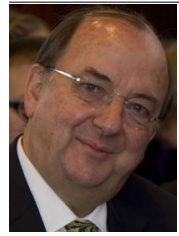

Prof. Dr. Dr. G. Gehrke

Klinik für Mund-, Kiefer- und

Gesichtschirurgie, plastische

und ästhetische Operationen

Diakoniekrankenhaus

Henriettenstiftung $\mathrm{gGmbH}$

Marienstraße 72-90

30171 Hannover

Gerd.Gehrke@ddh-gruppe.de

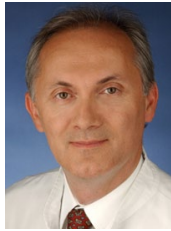

Prof. Dr. Dr. S. Reinert

Klinik und Poliklinik für Mund-, Kiefer- und Gesichtschirurgie

Universitätsklinikum Tübingen

Osianderstr. 2-8

72076 Tübingen

Siegmar.Reinert@

med.uni-tuebingen.de

\section{Den Hirntod darf nur ein Neurologe feststellen}

Jetzt ist es amtlich: Keine Hirntoddiagnostik ohne Neurologen oder Neurochirurgen. Um die Hirntoddiagnostik hat es zuletzt immer wieder Diskussionen gegeben, so sorgten Fälle für Aufsehen, in denen trotz unvollständiger Diagnostik bereits mit der Organentnahme begonnen worden war. Die neue Richtlinie zur Hirntoddiagnostik der Bundesärztekammer (BÄK) legt nun mehr Wert auf eine gute Qualifikation der Ärzte, die den Hirntod feststellen und den Körper zur Organentnahme freigeben dürfen. Zugleich finden bewährte apparative Methoden für den Nachweis des zerebralen Zirkulationsstillstandes wie Duplexsonographie und CT-Angiografie Eingang in die „Vierte Fortschreibung der Richtlinie zur Feststellung des irreversiblen Hirnfunktionsausfalls". Inhaltlich werden die Anforderungen an die Qualifikation der Ärzte präzisiert: Sie müssen nicht nur wie bisher über eine mehrjährige intensivmedizinische Erfahrung in der Behandlung von Patienten mit akuten und schweren Hirnschädigungen verfügen, sondern auch Fachärzte sein. Neu ist auch, dass mindestens einer der Ärzte, die den Hirntod feststellen, ein Facharzt für Neurologie oder Neurochirurgie sein muss. Bei Kindern bis zum vollendeten 14. Lebensjahr ist zusätzlich ein Facharzt für Kinder- und Jugendmedizin erforderlich. Ist dieser ein Neuropädiater, muss der zweite untersuchende Arzt kein Neurologe oder Neurochirurg sein. Neu ist auch, dass die Ärzte ihre Qualifikation gemäß der Richtlinie auf dem Protokollbogen bestätigen müssen. Den beteiligten Ärzten wird zudem eine regelmäßige Teilnahme an qualitätsfördernden Maßnahmen empfohlen. Diese sind jedoch keine Pflicht, auch fordert die BÄK keine entsprechenden Nachweise.
Kliniken obliegt Qualitätssicherung Die Richtlinie nimmt auch die Kliniken etwas stärker in die Pflicht. Sie müssen in einer Arbeitsanweisung festlegen, wie genau die Diagnostik zu verlaufen hat und dass diese gemäß der neuen Richtlinie erfolgt. Wie solche "Verfahren zur Qualitätssicherung der Todesfeststellung" im Einzelnen auszusehen haben, überlässt die Richtlinie aber den Kliniken.

www.aerztezeitung.de 


\section{Hier steht eine Anzeige.}

Springer 